\title{
Estilos de afrontamiento y salud mental en madres de niños con hipotiroidismo congénito
}

Pedro Luis Hernández González, ${ }^{1}$ Carmen Sánchez Pérez, ${ }^{1}$ Iván Rolando Rivera González ${ }^{1}$ y Leonor Sánchez Pérez ${ }^{2}$

\section{Introducción}

La enfermedad de un hijo representa una situación estresante ante la cual la madre responde de diversas maneras. A esta respuesta se le conoce como afrontamiento y puede esperarse en dos formas: haciendo frente al problema de manera directa (afrontamiento dirigido al problema) o aminorando el grado de trastorno emocional que se ha producido (afrontamiento dirigido a la emoción); estas respuestas pueden combinarse, pero predominará un estilo. En este proceso, la madre se ve influida tanto por factores personales como por factores situacionales; entre estos factores están el nivel de ansiedad, el grado de depresión, el apoyo social y el coeficiente intelectual (Lazarus y Folkman, 1986).

Entre las secuelas más severas del hipotiroidismo congénito $(\mathrm{HC})$, el retraso mental es la de mayor repercusión social;

1 Instituto Nacional de Pediatría.

2 Universidad Autónoma Metropolitana-Xochimilco. 
esta secuela se debe a la insuficiente o ausente producción de hormona tiroidea en un periodo crítico para la maduración del cerebro (Mayayo, 1995; Vela et al., 2004). Este padecimiento afecta a 1 de cada 3,500-4,000 niños nacidos vivos a nivel internacional. En México la prevalencia reportada en 2004 es de 1 caso por cada 2,574 recién nacidos tamizados (Canto-de Cetina et al., 1997; Vela et al., 2004; Velázquez et al., 1994).

El objetivo de este estudio consistió en describir los estilos de afrontamiento, medir algunas variables psicológicas con las que suelen estar vinculados y establecer las relaciones entre éstas, en un grupo de madres de niños con Hc detectados por tamiz metabólico y bajo tratamiento hormonal sustitutivo que asisten a un programa de seguimiento del neurodesarrollo para su evaluación.

\section{Método}

\section{Participantes}

El estudio se realizó en el Laboratorio de Seguimiento del Neurodesarrollo (LSND) del Instituto Nacional de Pediatría en donde se incluyeron a las madres de niños con HC detectados por tamiz, provenientes del Servicio de Endocrinología y que habían estado en seguimiento en el LSND. Se excluyeron a aquellas madres cuyos hijos tuvieran alguna enfermedad crónica además del HC 0 con antecedentes de otro hijo con Hc u otra enfermedad crónica.

\section{Material}

Inventario de modos de afrontamiento (ways of coping) de Lazarus y Folkman (1986), conformado por 67 ítems, cada uno con cuatro opciones de respuesta, dividido en ocho escalas que describen una serie de estilos cognitivos y comportamentales que las personas utilizan para dirigir las demandas internas y/o externas. En México, este instrumento ha sido validado y estandarizado (Ortiz, 1996; Sotelo y Maupome, 2000). 
Inventario de ansiedad rasgo-estado (IDARE) de Spielberger (1975). Este instrumento está formado por dos escalas independientes de autoevaluación para medir dos aspectos diferenciados: la ansiedad estado (A-E) y la ansiedad-rasgo (A-R). Se utilizó solamente la escala $A-R$ que comprende veinte reactivos con los que la persona puede indicar "cómo se siente en general".

Inventario de depresión de Beck. Es una escala autoaplicable que comprende 21 categorías de síntomas o actitudes, las cuales son cuantificadas con valores numéricos del 0 al 3; el puntaje obtenido indicará la intensidad de la depresión.

Instrumento no verbal de inteligencia Beta II-R, consta de seis subescalas. Se obtiene un coeficiente intelectual general (Kellogg y Morton, 2001).

Cuestionario de apoyo social de Sarason et al. (1983), el cual mide el apoyo percibido como disponible mediante 27 ítems. Cada ítem tiene dos partes: la primera evalúa el número de personas disponibles a quienes puede dirigirse el sujeto en momentos de necesidad, mientras que la segunda parte mide el grado de satisfacción de la persona con el apoyo percibido como disponible. Este instrumento ha sido validado en México (Mouriño y Viniegra, 1991).

\section{Procedimiento}

Se contactó a las madres de los niños, independientemente del tipo y edad de confirmación diagnóstica del Hc, se les explicó el motivo del estudio y se les invitó a participar mediante una carta de consentimiento informado. Los instrumentos se aplicaron de manera individual en dos sesiones, en la primera se emplearon el inventario de modos de afrontamiento (ways of coping) de Lazarus y Folkman; la escala ansiedad rasgo del IDARE de Spielberger, el inventario de depresión de Beck y el cuestionario de apoyo social de Sarason; haciéndoles resaltar que el interés se centraba en conocer cómo se sintieron durante todo el tiempo que han estado en el seguimiento. En la segunda sesión se aplicó el instrumento no verbal de inteligencia Beta II-R, y se recabaron datos sociodemográficos. A través de la historia clínica se obtuvo 
información sobre: el orden al nacimiento, tipo de hipotiroidismo, edad en días al inicio del tratamiento y los resultados del cociente intelectual del niño, aplicado por otro evaluador diferente al del estudio. Se utilizó estadística descriptiva para reportar las variables de las madres, los hijos y los instrumentos utilizados, se realizaron análisis de correlación con el coeficiente de Pearson tratando de establecer asociaciones, primero, entre factores maternos y los estilos de afrontamiento y, finalmente, entre los estilos de afrontamiento y las características del niño y del padecimiento. Se trabajó en el paquete estadístico JMP versión 8.

\section{Resultados}

Mediante muestreo no probabilístico por cuota se incluyeron 32 madres de niños con HC con un promedio de seguimiento de 7.1 años, cuyas edades oscilaron entre los 19 y los 52 años, con una edad media de $32.8 \pm 7.2$, y mediana de 33 años; al momento del estudio, $84 \%$ de las madres contaba con pareja, mientras que $16 \%$ restante no la tenía; $63 \%$ se dedicaban al hogar y $37 \%$ trabajaba; $72 \%$ contaba con una educación secundaria con promedio de años estudiados de $9.2 \pm 2.4$ y mediana de 9 .

Con respecto a los hijos se encontró $16 \%$ del sexo masculino y $84 \%$ femenino, por orden de nacimiento; $41 \%$, fue el primer hijo; 34\%, segundo; $13 \%$, tercero; y $12 \%$, cuarto o mayor; por tipo de hipotiroidismo, 53\% fueron atirósicos y 47\% nódulo sublingual; en cuanto al tratamiento, $25 \%$ lo habían iniciado antes o a los 30 días de edad; 44\%, entre 31 y 60 días de vida; y $31 \%$, después de esta edad; al momento del corte, el c.l. con puntaje mayor a 80 (promedio de $91.07 \pm 7.9$ y mediana de 89.6 ) correspondió a $78 \%$ del total de casos.

De las madres, $81 \%$ utiliza como principal estilo de afrontamiento el centrado en la emoción y $19 \%$ restante el dirigido al problema. El puntaje promedio en la escala de ansiedad fue de $42.8 \pm 8.8$ puntos, representando un bajo nivel de ansiedad. De la población, 56\% no presentó depresión, 19\% depresión leve, $16 \%$ moderada y 9\% severa. En el apoyo social se registró que 
$34 \%$ cuenta con dos personas como principal apoyo y todas tuvieron un grado de satisfacción positiva para el apoyo recibido. El c.I. materno reportó a 50\% de la población por arriba de 80 puntos (promedio $90.9 \pm 8.6$, mediana de 89.5 ), 34\% en rango de 79 a 70 puntos y $16 \%$ por abajo de 69 puntos.

De los factores asociados entre sí, referentes a la madre, se encontró correlación entre ansiedad y depresión r 0.64, y correlación negativa entre depresión y satisfacción del apoyo social $r-0.45$. Respecto de los estilos de afrontamiento maternos y los posibles factores asociados sólo se encontró correlación estadísticamente significativa $r 0.53$ con los puntajes de ansiedad. Entre los factores asociados con las variables de los hijos, se encontró correlación entre el c.ı. materno y el c.ı. de los niños r 0.35 y correlación negativa entre el c.l. del hijo y la depresión materna $r-0.35$. Entre los estilos de afrontamiento materno y las variables del niño no se encontraron correlaciones estadísticamente significativas.

\section{Discusión}

En este estudio, las madres de niños con $\mathrm{HC}$ bajo seguimiento utilizaron el afrontamiento dirigido a la emoción, coincidiendo con lo reportado para las enfermedades crónicas graves (Castillo, 1997; Miller et al., 2001; White et al., 1992); no podemos hacer comparación con la población en general porque no contamos con datos de población con este nivel de escolaridad y estrato socioeconómico. Factores como depresión, apoyo social y coeficiente intelectual no influyen de manera significativa en el estilo de afrontamiento materno utilizado ante la condición del hipotiroidismo y el c.I. del hijo, condiciones que a su vez tampoco mostraron asociaciones significativas con respecto al estilo de afrontamiento materno. La asociación de algunas variables con el c.ı. materno (independientes a los años de escolaridad materna), con elevada proporción de c.ı. bajo en rango de limítrofes e incluso de DM, obliga, al menos, a recomendar el tamiz con TSH a madres con niños diagnosticados como hipotiroideos. 


\section{Referencias}

Canto-de Cetina, T., Polanco, L., Ballote, Z.M., Fernández, G.F., Piña, M. y Cupul, D.G. (1997). Tamiz neonatal para hipotiroidismo congénito. Experiencia en el Laboratorio Regional del Sureste. Boletín Médico del Hospital Infantil de México, 54(8), 364-368.

Castillo, V. (1997). Estilos de afrontamiento de un grupo de madres de niños de 0 a 6 años con diagnóstico de retinoblastoma (tesis de licenciatura). Universidad Nacional Autónoma de México.

Kellogg, C. y Morton N. (2001). Instrumento no verbal de inteligencia BETA II-R. México: Manual Moderno.

Lazarus, R. y Folkman, S. (1986). Estrés y procesos cognitivos. Barcelona, España: Martínez Roca.

Mayayo, E. (1995). Hipotiroidismo. En O.J. Argente, L.A. Carrascosa, B.R. Gracia y H.F. Rodríguez (Eds.), Tratado de endocrinología pediátrica y de la adolescencia. (479-499). Madrid, España: Edimsa.

Miller, R., Sabin, C., Goldman, E., Clemente, C., Sadowski, H., Taylor, B. y Lee, C. (2001). Coping Styles in Families with Hemophilia. Psychological Health \& Medicine, 5(1), 3-12.

Mouriño-Pérez, R. y Viniegra-Velázquez, L. (1991). Diseño y validación de un instrumento para valorar el entorno psicosocial. Salud Pública de México, 33, 38-48.

Ortiz, R. (1996). Validación de un instrumento para medir afrontamiento. Tesis de especialidad. Universidad Nacional Autónoma de México.

Sarason, I., Levine, H., Basham, R. y Sarason, B. (1983). Assesing social support: The Social Support Questionnaire. Journal of Personality and Social Psychology, 44(1), 127139.

Sotelo, A.C. y Maupome, S.V. (2000). Traducción y estandarización del cuestionario modos de afrontamiento al estrés de Lazarus y Folkman para una población de adolescentes mexicanos. Tesis de licenciatura. Universidad Nacional Autónoma de México. 
Spielberger, C.D. (1975). IDARE inventario de ansiedad rasgo estado. México: Manual Moderno.

Vela, M., Gamboa, S., Loera-Luna, A., Aguirre, B.E., Pérez-Palacios, G. y Velázquez, A. (2004). Neonatal Screening for Congenital Hypothyroidism in Mexico: Experience, Obstacles, and Strategies. Journal of Medicine Screening, 6(2), 77-79.

Velázquez, A., Loera-Luna, A., Aguirre, B.E., Gamboa, S., Vargas, H. y Robles, C. (1994). Tamiz neonatal para hipotiroidismo y fenilcetonuria. Salud Pública de México, 36, 249-256.

White, N.E., Richter, J.M. y Fry, C. (1992). Coping, Social Support and Adaptation to Chronic Illness. Western Journal of Nursing Research, 14(2), 211-224. 
